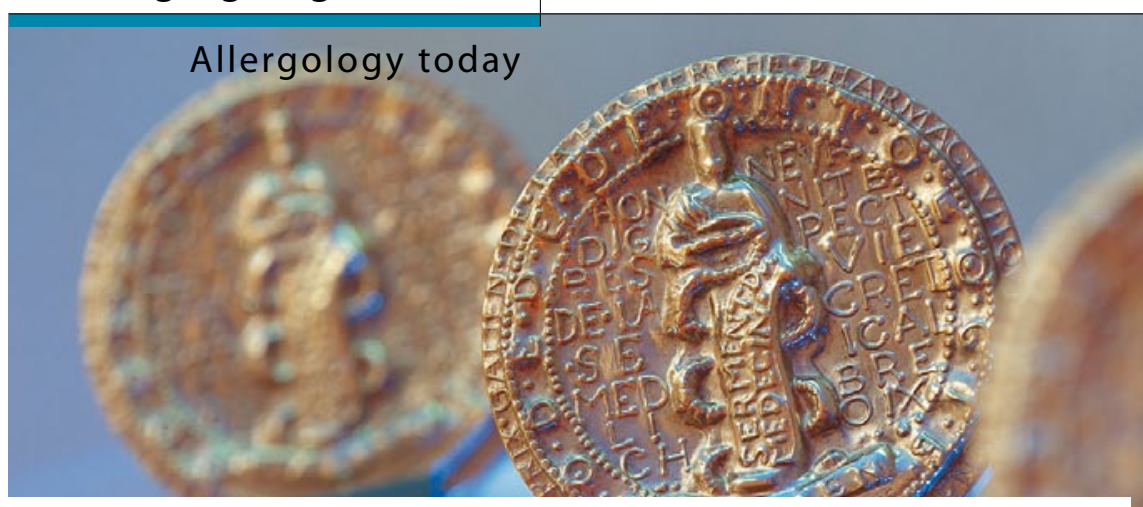

Galenus 2012

\section{Die Jury hat entschieden}

\author{
Er gilt als der inoffizielle "Nobelpreis" für Pharmakologie: der Galenus- \\ von-Pergamon-Preis. Am 18. Oktober wurde er in Berlin im Rahmen \\ einer Gala erneut verliehen - an drei beeindruckende Preisträger.
}

$\mathrm{M}$ it dem Galenus-von-PergamonPreis werden exzellente Grundlagenforschung in der Pharmakologie und herausragende pharmazeutische Innovationen ausgezeichnet. Die Preisträger 2012 sind Gilenya (Fingolimod) von Novartis Pharma in der Kategorie Primary Care und Zelboraf ${ }^{\oplus}$ (Vemurafenib) von Roche Pharma in der Kategorie Specialist Care. In der Kategorie Grundlagenforschung wurde das Team um Dr. Thomas Worzfeld aus Bad Nauheim für die Entwicklung eines neuen Ansatzes zur Therapie bei metastasierendem Brustkrebs geehrt.

Die 14-köpfige Jury unter dem Vorsitz von Prof. Dr. Erland Erdmann, Köln, tat sich bei ihrer Entscheidung schwer, wie der Jury-Präsident sagte. „Alle diesjährigen Kandidaten sind Innovationen." Mit Mehrheit hätten sich die Juroren schließlich für die diesjährigen Galenus-Gewinner entschieden. Wie wichtig Innovationen seien, betonte Harm van Maanen, Executive Vice President, Springer Medizin: „Innovationen sind eines der wichtigsten Güter, die wir haben." Deshalb sollten sie gefördert werden.

Fingolimod ist ein orales Medikament zur Therapie von Patienten mit Multipler Sklerose (MS). Es ist zugelassen für bisher nicht behandelte Patienten, die an einer rasch fortschreitenden, schwer schubförmigen MS erkrankt sind, sowie zur Eskalationstherapie, wenn trotz Behandlung mit einem $\beta$-Interferon eine hohe Krankheitsaktivität vorliegt. Mit Fingolimod gelingt es, die Schubrate zu verringern. Im Vergleich zur Behandlung mit Interferon ließ sich die
Schubrate in einer Zulassungsstudie um $52 \%$ senken ( 0,33 vs. 0,16 Schübe pro Jahr).

Vemurafenib ist die erste Option für eine personalisierte Therapie bei inoperablem oder metastasiertem Melanom. Das Medikament in Form von Filmtabletten ist zugelassen zur Therapie von Melanompatienten, deren Tumoren ein mutiertes BRAF-Gen aufweisen. In der Zulassungsstudie betrug die geschätzte mediane progressionsfreie Überlebenszeit 5,3 Monate. Im Vergleich: Bei Patienten, die Dacarbazin als Standardtherapie erhalten hatten, betrug sie nur 1,6 Monate. In der Vemurafenib-Gruppe war zudem das Sterberisiko um $63 \%$ und das Progressionsrisiko um $74 \%$ verringert. Die erste Phase der Entwicklung des Präparates erfolgte durch das US-Unternehmen Plexxikon.

Der Galenus-Preisträger Worzfeld und sein Team fanden heraus, dass der Rezeptor Plexin-B1 eine besondere Bedeutung für die Metastasierung bei Brustkrebs hat. Mammakarzinom-Patientinnen haben eine umso bessere Überlebenschance, je weniger Plexin-B1 exprimiert wird. Inzwischen wird ein monoklonaler Antikörper gegen diesen Rezeptor präklinisch getestet. „Anfangs dachten wir nicht, dass wir mit unserer Forschung bei Brustkrebs landen würden", so Worzfeld, der die Auszeichnung aus den Händen der Galenus-Vizepräsidentin Prof. Dr. Marion Kiechle, München, entgegennahm. Ausgangspunkt seien neurowissenschaftliche Fragestellungen gewesen. Geplant sind Untersuchungen mit dem Antikörper auch beim Magen- und beim Ovarialkarzinom.

Peter Leiner
CharityAward für Kinderhospiz Balthasar

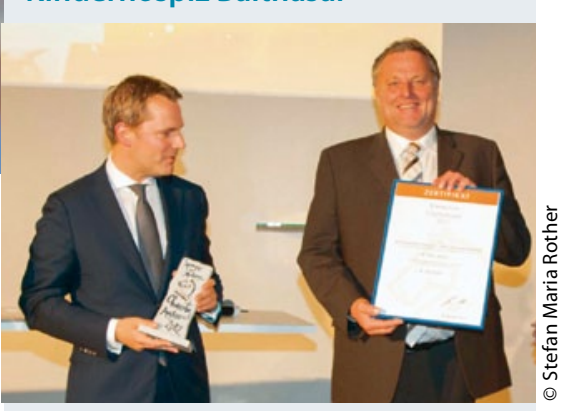

Auch der Springer Medizin CharityAward 2012 wurde am 18. Oktober verliehen. Ausgezeichnet wurde das Balthasar Kinder- und Jugendhospiz für sein unermüdliches Engagement. Träger des Hospizes ist die Gemeinnützige Gesellschaft der Franziskanerinnen zu Olpe. Schirmherr und Bundesgesundheitsminister Daniel Bahr überreichte den mit 150.000 Euro dotierten Preis.

Im Jahr 1998 gegründet ist Balthasar das erste Kinder- und Jugendhospiz in Deutschland. Dazu gehört auch Klartext!, eine Art „Sorgentelefon“. Es steht als Anlaufstelle für Jugendliche und junge Erwachsene zur Verfügung, die Situationen erleben, die durch Tod und Trauer geprägt sind. Die Unterhaltung einer solchen Einrichtung birgt immer wirtschaftliche Risiken, daher freut sich Hospizleiter Rüdiger Barth: „Wir sind stolz und glücklich über den Springer Medizin CharityAward für das Kinder- und Jugendhospiz Balthasar und klartext!. Durch das Medienpaket können wir unmittelbar Spenden generieren und zugleich auch neue Kontakte knüpfen, die vielleicht in eine langfristige Unterstützung münden. Eine doppelte Hilfe, für die wir all jenen danken möchten, die für uns gestimmt haben."

Der Springer Medizin CharityAward umfasst einen Barscheck über $50.000 €$ und ein Medienpaket über $100.000 €$. Mit dem Medienpaket unterstützt die Fachverlagsgruppe den Gewinner gezielt bei seiner Öffentlichkeitsarbeit. 